\title{
Hepatoprotective effects of metformin in hepatitis C virus-infected adolescents with beta thalassemia major
}

\author{
Ali Talebi ${ }^{1}$ and Mona Kargar ${ }^{1}$ \\ ${ }^{1}$ Tehran University of Medical Sciences
}

October 2, 2021

\section{Hepatoprotective effects of metformin in hepatitis C virus-infected adolescents with beta tha- lassemia major}

Dear Editor;

We read the recently published article by Abdel Monem et al. ${ }^{1}$ with great interest. The authors reported that metformin in antiviral naïve adolescents with beta thalassemia major ( $\beta-\mathrm{TM})$ infected with hepatitis $\mathrm{C}$ virus (HCV), can improve oxidative stress, as well as liver fibrosis, transaminases, and some symptoms. They proposed metformin as an option for its hepatoprotective effects in these patients until the initiation of antiviral agents.

Repeated transfusions expose patients with thalassemia to HCV infection ${ }^{2}$. Although cirrhosis and hepatocellular carcinoma are infrequent in children with this infection, in patients with thalassemia the presence of iron overload can increase the likelihood of progression of liver fibrosis ${ }^{3}$. So, the idea of using hepatoprotective agents in these patients seems appealing. Based on the importance of the issue, we think several points regarding the article are worth to be discussed.

Abdel Monem et al. included "HCV-infected $\beta$-TM patients" in their study. However, the diagnosis of $\mathrm{HCV}$ was based only on detecting anti-HCV antibody. Although spontaneous clearance of HCV infection in patients with thalassemia occurs less compared with patients without thalassemia, there is still a chance for it ${ }^{4}$. Hence, the interpretation of a positive HCV antibody test and differentiation of current infection from a resolved infection, or even a false-positive result ${ }^{5}$ is not possible. Indeed, it is emphasized to confirm the diagnosis of $\mathrm{HCV}$ in patients with positive anti-HCV antibody, with detecting viremia ${ }^{6}$.

Moreover, the study record in the registry of clinical trials raises questions regarding the inclusion criteria. The authors addressed the ClinicalTrials.gov registration number of NCT02984475. The inclusion criteria of the registered trial are patients with thalassemia regardless of their HCV serostatus. It is quite informative for the audiences if the authors elaborate on why the inclusion criteria have changed.

In the study, the score of liver stiffness and fibrosis grade were investigated by FibroScan. In table 3, changes in liver stiffness score for 30 patients in the control group and 27 patients in the metformin group were shown. However, for the fibrosis grade at baseline (table 1) and its changes (figure 4), only the data of 26 and 25 patients in the control and metformin group were presented, respectively. Since both parameters were assessed with the same scan, it was presumed to have the data of the same number of patients for liver stiffness score and fibrosis grade.

In the method section, it was mentioned that randomization of the patients into two groups was based on 1:1 allocation. Thus, within the 60 patients randomized, it was assumed to have two groups of 30 participants. However, the control and the placebo group included 31 and 29 patients, respectively. 
Among the participants, there were 12 patients with liver fibrosis of grade [?] 3 at baseline. Moreover, in 19 patients, the grade of liver fibrosis deteriorated at the end of the study. So, deciding to postpone the initiation of antiviral treatment seems to be discussable. The authors mentioned that during the recruitment after the approval of direct acting antivirals (DAAs) if patients "had the chance to receive antiviral treatment" they could leave the study. As shown in figure 1, there was only one patient who left the study for this reason. It is not clear what the chance of receiving antiviral treatment depended on. It should be noted that even before the era of DAAs, there was evidence supporting the treatment of patients with $\beta$-TM and HCV with pegylated interferon and ribavirin ${ }^{7-9}$.

Author Contributions: Both authors contributed in the concept and drafting the manuscript

Funding: None

Acknowledgements; None

Disclosures: The authors declare that they have no conflict of interest.

References:

1. Abdel Monem MS, Farid SF, Abbassi MM, et al. The potential hepatoprotective effect of metformin in hepatitis $\mathrm{C}$ virus-infected adolescent patients with beta thalassemia major: Randomised clinical trial. International Journal of Clinical Practice. 2021:e14104.

2. Daher HB, Sharara AI. Treatment of Chronic HCV Infection in Patients With Thalassemia. Clinical liver disease. 2019;14(6):199.

3. Spearman CW, Dusheiko GM, Hellard M, et al. Hepatitis C.Lancet . 2019; 394: 1451-1466

4. Kamal S, Abdelhakam S, Ghoraba D, et al. The course of hepatitis C infection and response to anti-viral therapy in patients with thalassemia major and hepatitis $\mathrm{C}$ infection: a longitudinal, prospective study. Mediterranean journal of hematology and infectious diseases. 2019;11(1).

5. Squires JE, Balistreri WF. Hepatitis C virus infection in children and adolescents. Hepatology communications. 2017;1(2):87-98.

6. Ghany MG, Morgan TR, panel AIhCg. Hepatitis C guidance 2019 update: American Association for the Study of Liver Diseases-Infectious Diseases Society of America recommendations for testing, managing, and treating hepatitis C virus infection. Hepatology.2020;71(2):686-721.

7. Di Marco V, Capra M, Angelucci E, et al. Management of chronic viral hepatitis in patients with thalassemia: recommendations from an international panel. Blood, The Journal of the American Society of Hematology. 2010;116(16):2875-2883.

8. Aminizadeh E, Alavian SM, Sari AA, Daryani NE, Behnava B. Safety and efficacy of adding ribavirin to interferon or peginterferon in treatment of hepatitis $\mathrm{C}$ infection in patients with thalassemia: a systematic review on randomized controlled trials. Hepatitis monthly.2016;16(3).

9. Borgna-Pignatti C, Gamberini MR. Complications of thalassemia major and their treatment. Expert review of hematology.2011;4(3):353-366. 